Available online @ https://jiem.jnnce.ac.in https:www.doi.org/10.37314/JJEM.2020.040104 Indexed in International Scientific Indiexing (ISI) Impact factor: 1.025 for 2018-19 Published on: 30 November 2020

\title{
Private Try-on Using Marker Based Augmented Reality
}

\author{
Shubham Singh Malik, Shiva Kumar R Naik, Vignesh S, Vidhatha A Shetty \\ School of Computing and Information Technology, REVA University, Bengaluru, India \\ R16CS527@cit.reva.edu.in, shivakumarnaik@reva.edu.in,R16CS472@cit.reva.edu.in, \\ R16CS471@cit.reva.edu.in
}

\begin{abstract}
Augmented reality is a possibility of providing us an environment where the objects are made to interact with the real-world environment. The objects are intensified using several techniques such as visual, audio, sense of touch, etc. The superimposition of the virtual objects on the real-world objects also help us to understand the computer's perception of space, along with how the humans understand the space. This paper provides a 3-D representation of apparel on our body using marker based augmented reality. This helps the user to get a better understanding of size and how it fits his/her body type instantly rather than placing an order and trying it out and returning it if it doesn 't fit properly.
\end{abstract}

Keywords: Augmented Reality, AR Tag, AR Toolkit, SLAM, Marker Detection

\section{INTRODUCTION}

Augmented Reality is now being one of the most talked about technologies since a long time. In the field of software development, it is among the leading technologies. It is now again gaining popularity with the release of products such as AR glasses, headsets, or smart phone technology. AR is a way to provide the real world information and to present it in such an interactive way such that the virtual elements can also be shown as the part of the real world. The superimposition of virtual objects in the field of view, can help us to reach out various possibilities, coupling the real and virtual worlds. It is a technology that uses recognition algorithms based on the computer vision. It can be simply understood by the example of a person who clicks an image from the real world; the underlying platform detects a marker. Then a virtual object is triggered to be superimposed on the top of the clicked image and the whole is displayed on the screen.

\section{Some fields where the Augmented Reality is used:}

Retail: The need of 'try it on' before purchasing clothes, shoes, glasses or anything that we could wear is made easier as never before. Brands like Gucci and Watch Box have made this luxury a new trend.

Construction \& Maintenance: Augmented Reality has made it possible for the architects, construction teams and patrons to visualize what the proposed design would look like. It helps them to understand the possible difficulties that may arise during construction.

Tourism: With the help of Augmented Reality solutions, the tourists can be provided with increased \& supplementary information and signspots to their destinations.

Education: The technological advancements in AR has helped the educators to provide the students a better interactive environment for learning with $3 \mathrm{D}$ models and more fun facts. 
Healthcare: The concept of Augmented Reality can be used to build digital images and critical information can be made accessible to the surgeons in 3D and within field of their view. It would help the surgeons to perform successful procedures without diverting away from the operating field to access crucial information required.

Navigation Systems: The safety of the navigation applications can be improved by the AR feature of Sygic. It combines with the smartphone's GPS with AR and then guides the driver along the virtual path. Navion has offered True AR. It is the first holographic based AR navigation system for cars.

There are two basic categories of AR:

I. Marker-based Augmented Reality: The distinct patterns that can be easily recognized and processed by the camera are known as markers. Markers can be uniquely identified and distinguished from the environment. Software that is usually developed in the form of an application, enables the user to scan the markers from the devices. After scanners such as a marker, an augmented experience can be triggered; it may be in the form of an object, text, or animation, to appear on the device.

II. Marker-less Augmented Reality: It is more versatile than the marker-based augmented reality. An image cue is not needed here to deploy. Device's camera, GPS, digital compass and the accelerometer provide the positional information, on which this form $A R$ relies upon. An understanding of 3D space is built using these inputs. It is achieved by the process of Simultaneous Localization and Mapping (SLAM). SLAM places the content directly to your field view of the world and sticks it to the environment.

\section{Literature survey}

[1] This paper projects a marker-based tracking system. The camera detects the QRCodes, then shows the media which is obtained from the server. Additionally, analyzation of such a system is also shown within the paper which is carried out by the breaking down the processing into pipelines stages.

[2] This paper provides a solution which makes it possible to acknowledge the vertices of any polygon having all the interior angles less than $180^{\circ}$ in an AR system. It uses linear programming theory.

[3] This paper proposes an approach where a customer will be able to see a 3D model of $\mathrm{him} / \mathrm{her}$, wearing garments on a personal device with the assistance of AR. Furthermore, it also provides the possibility to find the right size model.

[4] This paper talks regarding turning a standard real game board pawns into an AR game, with the assistance of a simple webcam and the Computer Vision techniques.

[5] This paper proposes a model based on learning to deduce the personalized $3 \mathrm{D}$ form of individuals. The proposed model uses two design approaches. The first, by predicting the form in T-pose space. The second relies upon the observations that the feed-forward predictions are fast but all the time do not align with the input images.

\section{Working of AR}

Augmented reality superimposes the video/image generated by the computer upon an image/video captured by the camera in such a way that the virtual objects appear to own specific locations within the real world. The working can be explained as:

A video/image from the real world is captured with the help of a device and it is fed to the computer. Once this is done the frames for the pre-placed markers are searched which principally comprise of quadrilaterals. This is done by the processing module. If an image is found, it will be then matched with the standard images of the markers. If we are able get a successful result out of the match, then it can be deduced that pre-placed markers are present in the scene captured. The vertices of the quadrilateral are considered while calculating the direction and position of the camera. Here optical centre of the camera is 
taken into account as the origin of coordinate system of the camera; and the centre of the marker is considered to be the origin of coordinate system of the word. The same positions as the marker's are used to draw the virtual figure. The output is given as the coupled image of the virtual figure and the real-world. The components through which it works can be given as:

Camera: It helps in streaming the footage of the real-world to the display and algorithm used for graphics.

Registration: The references of putting down the virtual objects is given by this step. Accelerometers, orientation sensors, barometric sensors and GPS are often more robust as compared to the high-contrast icons. Landmarks can even be thought of useful in providing orientation.

Computer Vision: The interpretation of the footage and also the manner of integrating the virtual object is determined by the computer vision. Some AR provide us with simply the relative placement over registration mark. Advanced algorithms can provide the shadows and motion.

Display: It can be any device such as smartphone or tablet that presents it back to the user. It can be thought as a union of the following technologies:

S.L.A.M: Simultaneous Localization and Mapping; it builds and updates the map of an unspecified environment, keeping the trail of the agent's position.

Depth Tracking: The distance of an object from the AR device is calculated using depth tracking.

Image Processing and Projection: The collection of the data and then its projection on a surface to view is done through the image processing and projection.

\section{Procedure for marker detection}

First step of the marker detection procedure is looking for the delineations of the potential markers, followed by the deduction of locations of marker's corners within the image. It is vital for the detection system to verify that it is really a marker and interpret its identity. Pose is calculated by the system using the knowledge from the detected marker location in the end. The basic procedure for marker detection can be stated using the steps mentioned below:

i) Acquisition of a high intensity image

ii) Preprocessing of the image (comprises of low-level image processing, appropriateness, detecting the lines and fitting them, detection of the corners of the marker)

iii) Detecting the most likely markers and discarding the conspicuous non-markers (quick rejection of the conspicuous non-markers and quick acceptance test for the most likely markers)

iv) Identifying and decoding the markers (template matching for template markers and decoding for data markers)

v) Calculating the marker pose (estimating the marker pose with repetitive pose calculation for the precise pose)

Due to its accessible source code AR Toolkit is one of the well-known systems for planar marking for Augmented Reality and Human Computer Interaction (HCI) systems. The bitonal markers accommodates of a black bordered square and a pattern in the interior of it. In variance to AR Toolkit, AR Tag markers with the edge-based approach, are such that an individual does not have to deal with the thresholds under different brightness conditions. Edge pixels found by an edge detector function are the basis for the marker detection process. They are coupled into segments that are then successively grouped into quadrangles. The algorithm proposed in this paper majority consists of these steps:

First the line segments are found by detecting edges on a coarse sampling grid and linking them along, so as to get longer lines, 
the line segments are integrated together. It is then followed by detection of all lines which are extended based on gradient information, so that we receive the lines of full length. Finally, the lines are sorted into quadrangles. To obtain quadrangles we forage for corner points by intersecting lines.

Then one line is picked from the set and the algorithm then tries to search out a corner by intersecting the chosen line with the right one out of the other lines present in the set. To find an appropriate second line several tests are carried out. The two lines must not be parallel or nearly parallel, as we tend to find quadrangular markers.

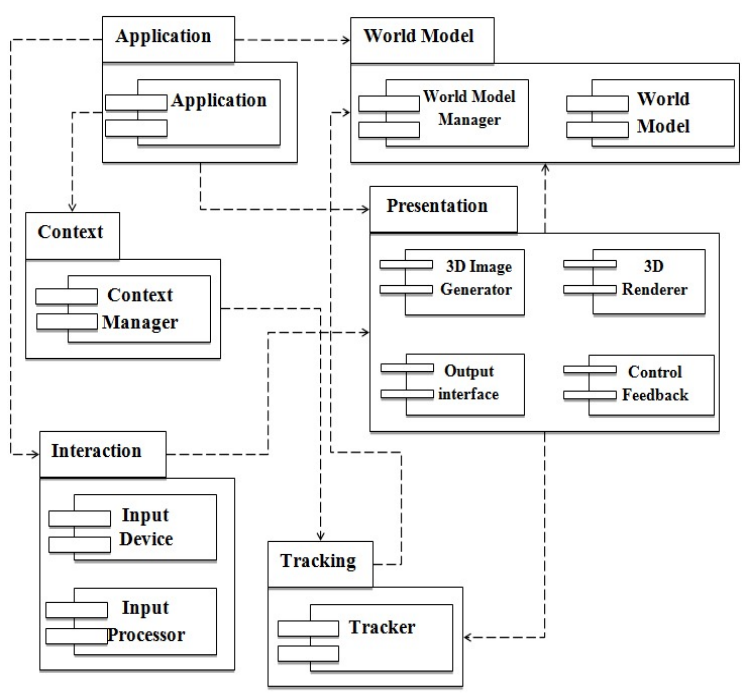

Figure 1: System Framework

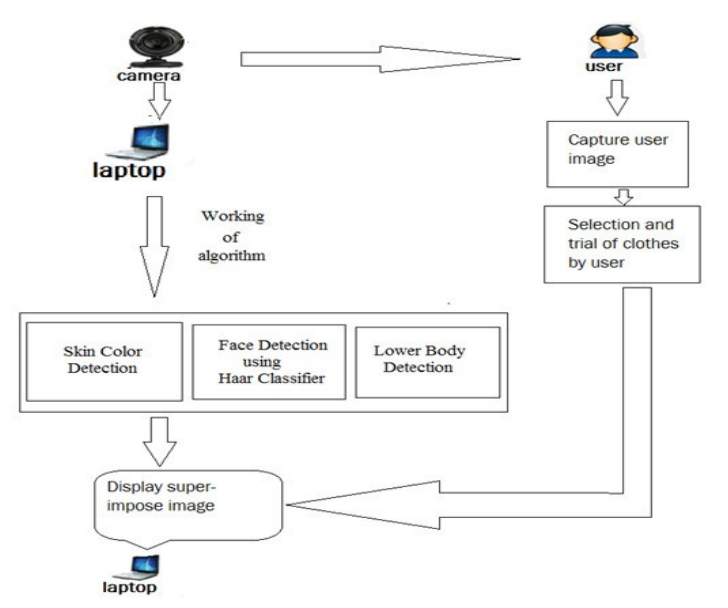

Figure 2: System Architecture
By doing so this algorithm overlooks extremely distorted markers wherever neighbouring sides are approximately parallel. However, this is often not an enormous issue since the interior of such markers cannot be sampled dependably anyway. Subsequently the next test checks the smallest distance between the two lines' end points.

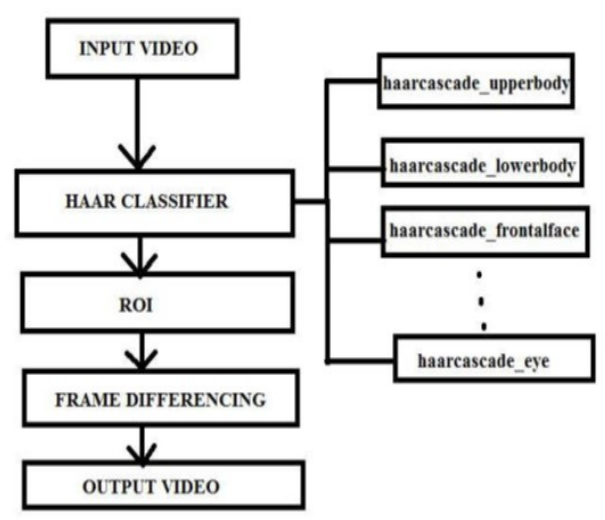

Figure 3: Flowchart for lower body detection

\section{Results}

As a result, through this approach with respect to the state-of-art we enhance the system in the below given aspect:

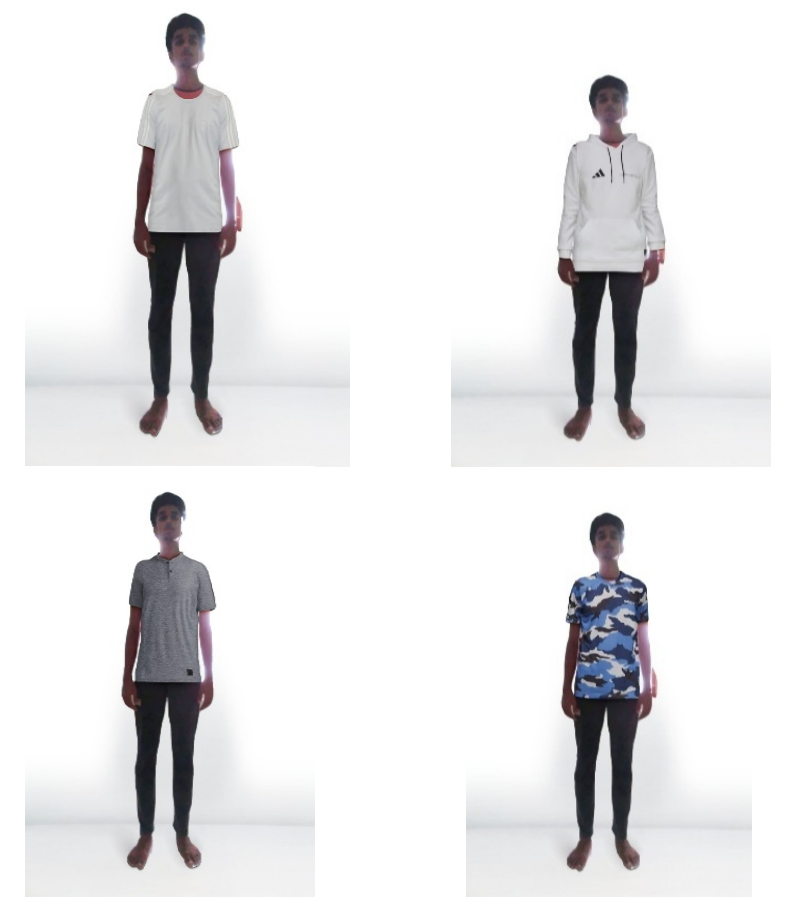

Figure 4: Virtually created apparel design augmented on user's body 
For the first time, the method proposed in this paper allows us to estimate the reconstruction of the full body of individuals in clothing, in a fully automatic manner. It can remarkably reduce the number of required images at the time of test. It can also compute the end result a several degrees faster than the state-of-art. The work in the future should enable the proposed method for scenarios where the subject may not be cooperating, for example from videos, or legacy movie material. Moreover, the clothes which do not fit with the geometry or are too far from the body that is clothes like skirts and coats; or the different hairstyles will be requiring a different and enhanced framework for more precise reconstructions and results.

\section{Conclusion}

The objective of this paper using marker based augmented reality is to provide virtual try-ons for buyers to watch themselves wear different garments without actually having to wear it physically. It allows a customer to vie a three-dimensional model of him/her wearing a particular garment in their smartphones. Such a system can improve a customers' shopping experience to make purchase decisions.

\section{References}

1. Alexandru Gherghina, Alexandru-Corneliu Olteanu and Nicolae Tapus," A Marker based Augmented Reality System" IEEE, 17-19 Jan. 2013, Sinaia, Romania.

2. Yan-Fei Gao, Heng-You Wang, XIAo-Ning Bian," Marker tracking for video based augmented reality ", Proceedings of the 2016 International Conference on Machine Learning and Cybernetics, Jeju, South Korea, 10-13 July, 2016.

3. Yoones A. Sekhavat, "Privacy Preserving Cloth Try-On Using Mobile Augmented Reality", IEEE, Transactions on Multimedia, Vol. 19, No. 5, May 2017.

4. Eray Molla, Vincent Lepetit," Augmented reality for Board Games", IEEE International Symposium on Mixed and Augmented Reality 2010 Science and Technolgy Proceedings 13 -16 October, Seoul, Korea.

5. Thiemo Alldieck, Marcus Magnor, Bharat Lal Bhatnagar, Christian Theobalt, Gerard Pons-Moll, "Learning to reconstruct people in clothing from a single RGB Camera". 